BRIEF REPORT

\title{
The effectiveness of television advertising campaigns on generating calls to a national Quitline by Māori
}

\author{
N Wilson, M Grigg, L Graham, G Cameron
}

Tobacco Control 2005;14:284-286. doi: 10.1136/tc.2004.010009

\begin{abstract}
Objective: To examine the effectiveness of four mass media campaigns on calls to a national Quitline by Māori (the indigenous people of New Zealand).

Methods: Monthly Quitline call data and calls within one hour of a television commercial (TVC) being shown were analysed for the 2002-2003 period. Data on target audience rating points (TARPs) and expenditure on TVCs were also used ( $n=2319$ TVC placements).

Results: Māori were found to register with the Quitline at higher rates during the most intense six campaign months (15\% more registrations compared to less intense months). The most effective campaign generated 115 calls per 100 TARPs by Māori callers within one hour of TVC airing (the "Every cigarette" campaign). A more Māori orientated campaign with both health and cultural themes generated 91 calls per 100 TARPs from Māori callers. For these two campaigns combined, the advertising cost per new registration with the Quitline by a Māori caller was \$NZ30-48. Two second hand smoke campaigns that did not show the Quitline number were much less effective at 25 and 45 calls per 100 TARPs.

Conclusions: These television advertising campaigns were effective and cost effective in generating calls to a national Quitline by Māori. Health authorities should continue to explore the use of both "threat appeal" style media campaigns and culturally appropriate campaigns to support Quitline use by indigenous peoples.
\end{abstract}

$\mathrm{N}$ ew Zealand's national Quitline has proved popular with the public, as indicated by the large number of calls to it. ${ }^{1}$ Call rates are known to be sensitive to media publicity on smoking and health risks, ${ }^{2}$ and even to international events. ${ }^{3}$ There is also evidence that this service enhances quit rates among its users. ${ }^{4}$ The Quitline service attempts to reach all smoking adults, but has a particular focus on supporting quitting by Māori smokers. Māori comprise $15 \%$ of the New Zealand population and $49 \%$ of Māori adults smoke (around 190000 smokers). This high smoking rate contributes to lower life expectancy for Māori and increases national inequalities in health. ${ }^{5}$ To explore further the use of this service by Māori, this study examined the impact of four recent mass media campaigns.

\section{METHODS}

\section{The campaigns}

The "Every cigarette is doing you damage" (EC) campaign uses "threat appeal" themes on the adverse health consequences of smoking and also themes on the promotion of quitting. Although developed in Australia, some of the television commercials (TVCs) have been adapted to include New Zealand and Māori specific content.
The "Its about whānau" (IAW) campaign was designed by Māori specifically for a Māori audience. It uses personal testimonials with themes that include the promotion of quitting and being smoke-free for health and to protect whānau (family). As with the EC campaign, all the TVCs show the Quitline number and further details are described more fully elsewhere. ${ }^{67}$

Two other campaigns both covered secondhand smoke (SHS) themes. The "World Smokefree Day" (run in May 2003) and the "Lets Clear the Air" campaign (run for six months in 2003).

\section{Study period selection}

The focus of this analysis was on the years 2002 and 2003, since this allowed for comparison of both the EC and IAW campaigns after the latter had got past the initial launch period (it was launched in August 2001). The data for the preceding years were considered to be of less value for studying EC campaign effectiveness since calls to the Quitline in 2000 and 2001 were thought to be particularly influenced by callers wishing to gain access to heavily subsidised nicotine replacement therapy (patches and gum), which was introduced in November 2000.

\section{Exposure and response data}

The target audience rating points (TARPs) for each TVC were collected ( $\mathrm{n}=2319$ TVC placements). These reflected the estimated television advertising exposure for those in the 2544 year age group who were in the three most deprived of six socioeconomic groupings. The TARPs were adjusted to reflect the proportion of Māori in this age group (that is, $14.7 \%$ ).

Data on the number of calls by Mãori to the Quitline within one hour of each TVC being shown was analysed for 1482 TVC placements (table 1). This dataset excluded the 631 TVC placements that were not shown at the national level (that is, at just a regional level) and TVC placements for which ethnicity data for callers was not available (that is, $12 \%$ of the remaining calls). Data on the total calls to the Quitline's call centre and new registrations to the Quitline were also obtained (that is, where callers register for obtaining structured Quitline support and advice in making a quit attempt).

\section{Attribution of impact to the TVCs}

Those callers registering with the Quitline were asked about their source of Quitline information, such as friends, family, and media advertising. These data were used in the cost effectiveness calculations. The minimum level of attribution to television advertising was $41 \%$ since this is the proportion of those who explicitly state "television advertising" as the source of information about the Quitline. ${ }^{8}$ The maximum

Abbreviations: EC, "Every cigarette is doing you damage"; IAW, "Its about whānau"; SHS, secondhand smoke; TARPs, target audience rating points; TVC, television commercial 
Table 1 Impact of various campaigns and television commercials (TVCs) for generating calls to the New Zealand national Quitline from Māori callers (for calls within one hour of showing a TVC)

\begin{tabular}{|c|c|c|c|c|c|}
\hline Campaign/TVC & $\begin{array}{l}\text { Total TVC } \\
\text { placements }\end{array}$ & $\begin{array}{l}\text { Total calls } \\
\text { within } 1 \text { hour }\end{array}$ & $\begin{array}{l}\text { Total } \\
\text { TARPs* }\end{array}$ & $\begin{array}{l}\text { Calls per } 100 \\
\text { TARPs }\end{array}$ & $\begin{array}{l}\text { Rate ratio } \\
(95 \% \mathrm{Cl})\end{array}$ \\
\hline \multicolumn{6}{|c|}{ Campaigns that used the Quitline number } \\
\hline "Every cigarette" & 372 & 383 & 333 & 115 & $1.26(1.08$ to 1.46$)$ \\
\hline "Its about whānau" (family) & 483 & 439 & 480 & 91 & Reference \\
\hline \multicolumn{6}{|c|}{ Campaigns that did not use the Quitline number } \\
\hline "World Smokefree Day" & 134 & 35 & 78 & 45 & 1.79 (1.17 to 2.73$)$ \\
\hline "Lets Clear the Air" & 493 & 82 & 328 & 25 & Reference \\
\hline
\end{tabular}

*Target audience rating points: a measure of estimated exposure of the target audience.

$\mathrm{Cl}$, confidence interval.

level was estimated to be $65 \%$ and was based on additional attribution to TVCs via indirect means (for example, via assigning $50 \%$ of the "friends and family" reported source of information to TVCs-as detailed more fully elsewhere ${ }^{7}$ ). The proportion of campaign expenditure (IAW and EC combined) attributed as being orientated towards a Māori audience was that of the proportion of Māori in the group for which the TVCs were most targeted (that is, $14.7 \%$ in the $25-44$ year age group). This was a simplistic approach as only $53 \%$ of all callers were from this age group and the IAW campaign was also more weighted towards Māori.

\section{RESULTS}

\section{Monthly call data}

The relatively intense six campaign months (that is, those with over 480 TARPs/month) were compared with the other 18 months. In these "intense" months, Māori callers registered with the Quitline at a $15.2 \%$ greater rate (866 per month average versus 735 for total registrations).

\section{Campaign effectiveness}

Based on calls to the Quitline within one hour of a TVC airing, the most effective campaign for generating calls by Māori was the EC campaign at 115 calls per 100 TARPs (table 1). It was 1.3 times more effective in generating calls than the IAW campaign at 91 calls per 100 TARPs. While the two campaigns relating to SHS also generated calls to the Quitline, they were much less effective than the other two campaigns.

\section{Cost effectiveness}

Over this two year time period, there were 15486 new Māori callers registered with the Quitline $(21.3 \%$ of all new registrations, and an estimated $8.2 \%$ of all Māori adult smokers). Also the attributed television advertising expenditure directed at Māori was estimated to be \$NZ304 560. The advertising cost per new registration by a Māori caller was estimated to be $\$ 30$ to $\$ 48$ (as per the attribution method detailed in the methods section).

\section{DISCUSSION}

\section{Main findings}

This study found that television advertising campaigns are successful in generating calls to a national Quitline from indigenous New Zealanders. Effectiveness was highest for those campaigns in which the Quitline number was included in the TVCs, and for the particular campaign that had both "threat appeal" and "calling for help" components (the EC campaign). Two of the four TVCs in this campaign were modified to include Māori specific aspects, and so these may have contributed to its effectiveness for Māori. Although the more Māori orientated campaign (IAW) was less effective in generating calls than the EC campaign, it is not strictly comparable as it covered a much broader scope of health issues (including smoking in pregnancy and SHS). It also had the broader theme of affirming Māori cultural identity, which has potentially both social and health benefits.

These findings are consistent with other data indicating that the IAW campaign was regarded favourably by a Māori audience, ${ }^{910}$ and with data on Quitline usage for the nonMāori and Pacific peoples in New Zealand. ${ }^{\text {? }}$

The cost effectiveness data suggests that expenditure on television advertising smoking cessation campaigns is a good use of public health sector expenditure. Even so, this effectiveness could be greatly enhanced if tobacco control in New Zealand was further strengthened (for example, by raising tobacco taxes).

\section{Study limitations}

This study compared campaigns which had somewhat different objectives. Two campaigns consistently used the Quitline number in all TVCs while the two SHS campaigns did not include the number and did not have Quitline calls as an objective at all. Another difference between the campaigns was that the EC campaign had been running for over two years longer than the IAW campaign (albeit with some variation in the TVCs used).

During some of the year 2003, both the EC and IAW campaign TVCs were placed in time slots outside of Quitline opening hours as part of "general awareness raising" rather than to generate Quitline calls. This was to reduce the risk of the Quitline capacity being overloaded, to support and remind people undertaking quit attempts, and to support those who may choose to quit without the Quitline assistance. However, the probable outcome of this approach is that the results for calls per TARPs reported here are likely to be underestimates, relative to the use of other TVC placement strategies focused entirely on attracting Quitline calls. Even so, some of these calls to the Quitline may also be motivated by factors additional to the content of the TVCsfor example, access to the vouchers for heavily subsidised nicotine replacement therapy that are provided by the Quitline. Other more minor limitations with this study are detailed more fully elsewhere. ${ }^{7}$

\section{What this paper adds}

This study examined the impact of four television advertising campaigns on calls to a national Quitline service by Māori (New Zealand's indigenous population). The two campaigns with a Quitline telephone number were effective and cost effective in generating calls (particularly the one with the threat appeal messages). Health authorities should continue to support and further develop such campaigns and Quitline services to protect the health of indigenous peoples and reduce health inequalities. 


\section{Implications for policymakers}

Given this evidence for effectiveness and cost effectiveness, health authorities should continue to invest in such television campaigns and national quitline services. TVCs that are borrowed from other countries (as per the EC campaign) can be cost effectively utilised for different populations (at least for certain threat appeal messages). Nevertheless, local adaptation of these TVCs to maximise appropriateness for an indigenous audience is desirable so as to ensure a reduction in health inequalities attributable to smoking. Complete local development of TVCs by indigenous health workers for their own population may be more expensive, but it has the potential benefit of maximising authenticity and cultural appropriateness of the TVCs. As television channels multiply (for example, the new Māori television channel) there will be even greater scope for expanding reach to indigenous peoples and therefore maximising the benefits to health and the reduction of health inequalities.

\section{ACKNOWLEDGEMENTS}

The authors wish to thank the Quitline callers who provided information about their ethnicity. They also thank Ramzan Afzal, Helen Glasgow, and Dr George Thomson who provided useful comments on the draft. This study was funded by The Quit Group which runs the Quitline on contract to the New Zealand Ministry of Health.

\section{Authors' affiliations}

N Wilson, Wellington School of Medicine \& Health Sciences, Wellington, New Zealand

M Grigg, G Cameron, The Quit Group, Wellington, New Zealand L Graham, Graham Strategic, Wellington, New Zealand
Competing interests: none declared

Correspondence to: Dr Nick Wilson, Wellington School of Medicine \& Health Sciences, PO Box 7343, Wellington, New Zealand; nwilson@ actrix.gen.nz

Received 17 September 2004

Accepted 20 January 2005

\section{REFERENCES}

1 Grigg M, Glasgow H. Subsidised nicotine replacement therapy. Tobacco Control 2003; 12:238-9.

2 Wilson N, Hodgen E, Mills J, et al. Journal article on smoking and blindness prompts significantly more calls to the Quitline. N Z Med J 2002;115:199-200.

3 Wilson N, Hodgen E, Mills J, et al. Events of 11 September 2001 significantly reduced calls to the New Zealand Quitline. Tobacco Control 2002:11:280.

4 BRC. Evaluation of subsidised NRT exchange card scheme and the Quitline subsidised NRT exchange card programme. Wellington: BRC Marketing \& Social Research, May, 2004.

5 Tobias M, Cheung J. Inhaling inequality: tobacco's contribution to health inequality in New Zealand, (Public Health Intelligence Occasional Bulletin No.7). Wellington: Ministry of Health, 2001, http://www.moh.govt.nz/ moh.nsf/ea6005dc347e7bd44c2566a40079ae6f/ eb38a31 c067f8776cc256af0000f6fle/\$FILE/Inhalinglnequality.pdf (Accessed 28 August 2004).

6 The Quit Group. Quit campaigns. http://www.quit.org.nz/ page.php3? $=70$ (Accessed 28 August 2004).

7 Wilson N. The impact of television advertising campaigns on calls to the New Zealand Quitline. Wellington: The Quit Group, 2004, http:// www.quit.co.nz/documents/FINALMediaStudy.pdf.

8 The Quit Group. Quitline calls analysis report July 2002 to June 2003. Wellington: The Quit Group, 2003.

9 Waa A, Grigg M. "Its about whanau": Views of smokers and whanau. In: Proceedings of the Tobacco Control Research Symposium, September 2002. Wellington: Health Sponsorship Council, 2003:19-21.

10 Barnes HM, McPherson M. Maori smoker and whanau response to "It's about whanau" television commercials. Auckland: Whariki Research Group, SHORE Research Centre, Massey University, 2003.

\section{The Lighter Side}

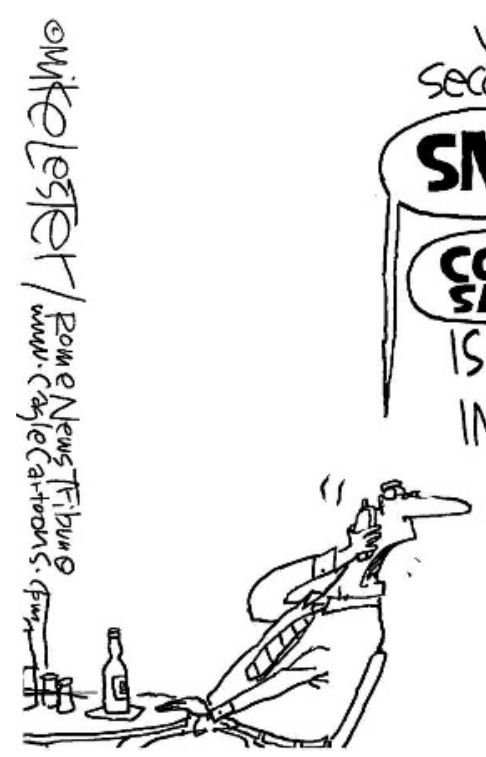

YOUR SECOND-HAND

SMOKE
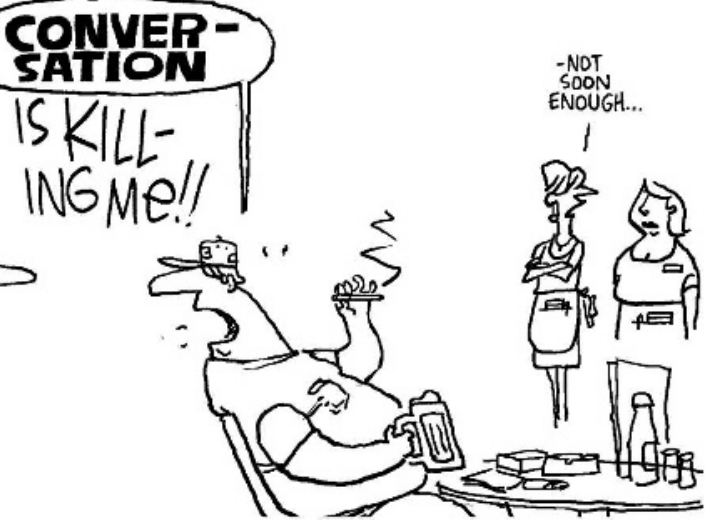

(c) Mike Lester. Rome News Tribune, www.caglecartoons.com. 